\title{
Rare Aggressive Form of Multiple Myeloma with Diffuse Osteosclerosis and Elevated CA 15-3 Levels
}

\author{
Dally Najib ${ }^{1,2}$, Daniel Talmud ${ }^{3}$, Saher Srour ${ }^{2,4}$, Rola Khamisy-Farah5 ${ }^{5}$ Raymond Farah ${ }^{1,2 *}$ \\ ${ }^{1}$ Department of Hematology, Ziv Medical Center, Zefat, Israel \\ ${ }^{2}$ Faculty of Medicine in the Galilee, Bar-Ilan University, Safed, Israel \\ ${ }^{3}$ Department of Internal Medicine, Ziv Medical Center, Zefat, Israel \\ ${ }^{4}$ Department of Radiology, Ziv Medical Center, Zefat, Israel \\ ${ }^{5}$ Clalit Health Service, Akko, Israel \\ Email: "Raymond.F@ziv.health.gov.il
}

Received 30 January 2016; accepted 22 March 2016; published 25 March 2016

Copyright (C) 2016 by authors and Scientific Research Publishing Inc.

This work is licensed under the Creative Commons Attribution International License (CC BY).

http://creativecommons.org/licenses/by/4.0/

(c) () Open Access

\section{Abstract}

We report the case of a 56-year-old female who had recently been examined for back and epigastric pain, and was diagnosed in the internal medicine ward as having an aggressive rare form of multiple myeloma with diffuse osteoblastic bone lesions. She also had high levels of breast tumor marker (CA 15-3), severe tumor lysis syndrome, and pleural and central nervous system involvement. A few cases of multiple myeloma with diffuse osteosclerosis that are not part of POEMS syndrome (polyneuropathy, organomegaly, endocrinopathy, monoclonal gammopathy, skin changeshave) have been described. None of these cases were as aggressive as our case. To our knowledge it is the first report describing an elevation in CA 15-3 in conjunction with multiple myeloma.

\section{Keywords}

Multiple Myeloma, Osteosclerosis, CA 15-3, Pleural Effusion

\section{Introduction}

Multiple myeloma is the prototype of plasma cell tumor. The patients usually present with anemia, renal failure, hypercalcaemia or lytic bone lesions. On rare occasions multiple myeloma may be associated with osteosclerosis. According to Kyle et al. osteoblastic lesions are rare manifestations of MM ( $0.5 \%$ of patients) [1]. Most of

\footnotetext{
${ }^{*}$ Corresponding author.
}

How to cite this paper: Najib, D., Talmud, D., Srour, S., Khamisy-Farah, R. and Farah, R. (2016) Rare Aggressive Form of Multiple Myeloma with Diffuse Osteosclerosis and Elevated CA 15-3 Levels. Open Journal of Internal Medicine, 6, 11-14. 
the time, it occurs in conjunction with POEMS syndrome (Polyneuropathy, organomegaly, endocrinopathy, monoclonal gammopathy and skin changes). We present the case of a 56-year-old female who was diagnosed with multiple myeloma with diffuse osteosclerosis and elevated CA 15.3 levels.

POEMS syndrome is a rare multisystemic disease that occurs in the setting of a plasma cell dyscrasia. The pathophysiologic link between the constellation of symptoms and the underlying disease is not well understood, but the link may be related to changes in the levels of a cytokine or a growth factor.

\section{Case Report}

A 56-year-old female was presented to the internal medicine ward with epigastric, back and flank pain. She also reported alternating fever and weight loss for a 2 -week period. She denied vomiting, shortness of breath or any urinary symptoms. Her past medical history was positive only for uncomplicated duodenal ulcer, which is treated with Proton pump inhibitor. On admission the patient was afebrile, normotensive with normal cardiac rhythm. Epigastric and flank tenderness was noted on her examination, with no signs of peritonitis. The physical examination was otherwise normal. Complete blood count showed leukocytosis $(14,600 / \mu \mathrm{L})$ and mild thrombocytopenia $(80,000 / \mu \mathrm{L})$. The hemoglobin level initially was normal-13.6 g/dl. Blood chemistry showed normal level of sodium (141 meq/l) and potassium (3.8 meq/l). Serum creatinine was $0.99 \mathrm{mg} / \mathrm{dl}$, BUN $14.3 \mathrm{mg} / \mathrm{dl}$, total bilirubin $0.33 \mathrm{mg} / \mathrm{dl}$. AST was $100 \mathrm{IU} / 1$, ALT $32 \mathrm{IU} / 1$, GGT $363 \mathrm{IU} / 1$, Alk-Phos $166 \mathrm{IU} / 1$, and a significant elevation of LDH was noted $2388 \mathrm{IU} / \mathrm{l}$. CRP was also elevated $59 \mathrm{mg} / \mathrm{dl}$. There was mild hyperproteinemia, with a total protein of $8.93 \mathrm{~g} / \mathrm{dl}$, and normal level of albumin $(3.9 \mathrm{~g} / \mathrm{dl})$ and calcium $(9.9 \mathrm{mg} / \mathrm{dl})$. The level of the tumor marker CA 15.3, which is known to be elevated in cases of breast cancer, was $635 \mathrm{U} / \mathrm{ml}$, more than 20 -fold the upper normal limit. Peripheral blood smear was normal. On CT scan of the chest, abdomen and pelvis there were multiple sclerotic lesions in the skeleton, including the vertebras, ribs, sternum, pelvic bones and femurs (Figure 1). A SPECT bone scan revealed pathologic uptake in seven vertebras, femurs, sternum, clavicles and three ribs (Figure 2). These findings suggest diffuse osteoblastic lesions. The urine was positive for Bence Jones protein. Serum electrophoresis showed a monoclonal paraprotein band of $3.1 \mathrm{~g} / \mathrm{dl}$ and elevated free kappa light chain in the blood. Immunofixation was positive for IgA kappa. A bone marrow biopsy was preformed and confirmed the diagnosis of multiple myeloma with more than $50 \%$ plasma cells. Cytogenetic evaluation revealed $17 \mathrm{p}(\mathrm{p} 53)$.

To rule out the possibility of breast malignancy that may cause osteoblastic bone lesions, the patient underwent a breast ultrasonography and mammography with normal findings. After hematologic consultation, the patient initiated therapy with VCD protocol which contains Bortezomib (proteosome inhibitor), cyclophosmamide and corticosteroids. Shortly after treatment was started, severe tumor lysis syndrome occurred. The patient was treated appropriately with intravenous fluids and allopurinol until her condition was stabilized and she showed improvement on her kidney function tests and electrolyte levels. One month later the patient suffered from dyspnea. Chest X-ray and CT scan showed a massive left pleural effusion. A pleural drain was inserted and histological and flow cytometric evaluation showed plasma cells in the pleural fluid. The patient was transferred to a tertiary center in Haifa due to severe dyspnea and pleural effusion that is untreatable by drainage only. There,

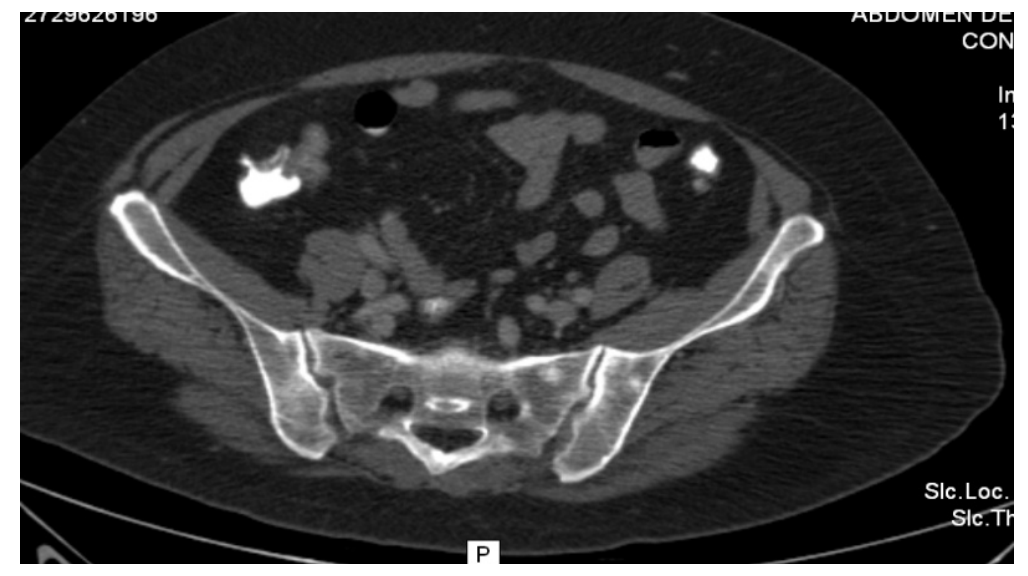

Figure 1. Axial CT scan showing sclerotic lesions at the sacrum and the ilium. 


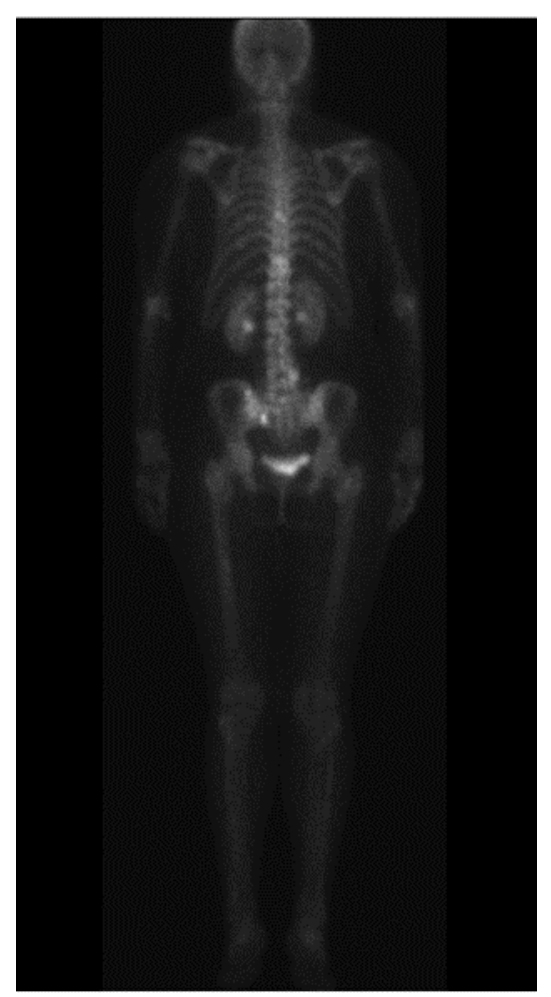

Figure 2. SPECT bone scan showing pathologic uptake in the vertebras, sternum and ribs.

she started feeling numbness around her lips. A lumbar puncture was done and myeloma cells were found in the CSF. There was no sign of leptomeningial involvement on MRI.

\section{Discussion}

There are three unique elements in our patient: 1) diffuse osteoblastic lesions; 2) extremely high levels of CA15.3 and 3) unusually aggressive behavior of the disease with rare organ involvement and tumor lysis syndrome.

There are four subtypes of osteoscelerosis in myeloma: 1) diffuse; 2) focal osteocondensation; 3) bony spiculation on the surface of the bone and 4) sclerotic reaction at the rim of lytic lesion. Sclerotic lesions usually affect men more than women (4:1 ratio as opposed to $1: 1$ in all cases of $\mathrm{MM}$ ). There is no difference in the prognosis [2].

The entity of diffuse osteosclerosis must be distinguished from the osteosclerosis associated with POEMS syndrome. With the latter, the lesions are usually small, isolated and not painful. Our patient had none of the above mentioned manifestations of POEMS syndrome. In 1997, Lacy et al. reviewed 15 cases of MM with diffuse osteoblastic lesions not related to POEMS syndrome [3]. Another case was described in 2012 [4]. The most common presenting symptoms were bone pain and anemia. None of these reports mentioned pleural and central nervous system involvement or tumor lysis syndrome like we had in this case. The exact pathogenesis of osteosclerosis is not known. It is most likely derived from greater activity of osteoblasts, as compared to osteoclasts.

CA 15-3 is a mucin that is encoded by the MUC1 gene, and is expressed by normal and malignant epithelial cells. It serves as a tumor marker for breast cancer. CA 15-3 can predict the pre-operative and overall prognosis of primary breast cancer, as well as the possibility of metastasis [5]. Literature review did not found a connection between CA 15-3 and multiple myeloma.

\section{Conclusion}

Our case is an interesting unique case that describes a rare manifestation of aggressive multiple myeloma with osteoblastic bone lesion and high level of tumor marker. The case can help us think about diagnosis or exclusion 
of multiple myeloma especially when we meet a patient describing these rare presentations as in the our described case.

\section{References}

[1] Kyle, R.A., Gertz, M.A., Witzig, T.E., Lust, J.A., et al. (2003) Review of 1027 Patients with Newly Diagnosed Multiple Myeloma. Mayo Clinic Proceedings, 78, 21-33. http://dx.doi.org/10.4065/78.1.21

[2] Blaquière, R.M., Guyer, P.B., Buchanan, R.B. and Gallagher, P.J. (1982) Sclerotic Bone Deposits in Multiple Myeloma. The British Journal of Radiology, 55, 591-593. http://dx.doi.org/10.1259/0007-1285-55-656-591

[3] Lacy, M.Q., Gertz, M.A., Hanson, C.A., Inwards, D.J. and Kyle, R.A. (1997) Multiple Myeloma Associated with Diffuse Osteosclerotic Bone Lesions: A Clinical Entity Distinct from Osteosclerotic Myeloma (POEMS Syndrome). American Journal of Hematology, 56, 288-293.

http://dx.doi.org/10.1002/(SICI)1096-8652(199712)56:4<288::AID-AJH16>3.0.CO;2-5

[4] Patoliaa, S., Schmidta, F., Patoliaa, S., Gulati, N., Muhammad, P., Narendra, D., Enriquez, D. and Quist, J. (2012) Multiple Myeloma with Mixed Lytic and Blastic Bone Lesions with Lymphadenopathy: Rare Manifestation of a Common Disease-Case Presentation and Literature Review. World Journal of Oncology, 3, 78-82. http://dx.doi.org/10.4021/wjon $440 \mathrm{w}$

[5] Shering, S.G., Sherry, F., McDermott, E.W., O’Higgins, N.J. and Duffy, M.J. (1998) Preoperative CA15-3 Concentrations Predict Outcome of Patients with Breast Carcinoma. Cancer, 83, 2521-2527. http://dx.doi.org/10.1002/(SICI)1097-0142(19981215)83:12<2521::AID-CNCR17>3.0.CO;2-A 